\title{
Regards
}

\section{Michel Sebillotte, agronome : penser l'action}

\section{Propos recueillis par François Papy}

\author{
Michel Sebillotte ${ }^{1}$, François Papy ${ }^{2}$ \\ 1 Agronome, professeur émérite de l'INA P-G, ancien directeur scientifique à l'INRA \\ 2 Agronome, ancien directeur de recherche à l'INRA, 92 avenue Victor Hugo, 92170 Vanves, France
}

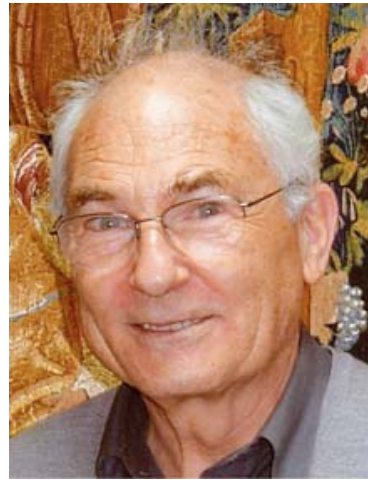

(Droits réservés)

\begin{abstract}
Michel Sebillotte, décédé le 7 avril 2010, a fortement marqué l'évolution de l'agronomie en France. Nous publions ici un entretien qu'il a donné à notre revue juste avant la longue maladie qui l'a emporté. Titulaire de la chaire d'agronomie de l'INA P-G et directeur de ses laboratoires de recherche jusqu'en 1993, Michel Sebillotte a formé de nombreuses générations d'ingénieurs agronomes. Spécialiste des systèmes de culture et de la fertilité $\mathrm{du}$ milieu, il propose le concept $\mathrm{d}^{\prime}$ «itinéraire technique » pour l'analyse des pratiques culturales des agriculteurs et la conception de nouveaux procédés de culture pour une agriculture durable. Plus tard, afin d'analyser les décisions à l'origine des pratiques des agriculteurs, il crée le concept de «modèle pour l'action ». Détaché, en 1993, à la direction de l'Inra, il est chargé de créer un service de prospective et formalise une méthodologie appropriée aux organismes de recherche, la méthode SYSPAHMM (SYStème-Processus-Agrégats d'Hypothèses-Micro-Macroscénarios). Afin de répondre aux problèmes que rencontrent les acteurs régionaux, il conçoit et anime un dispositif interdisciplinaire de recherche en partenariat : le programme «Pour et sur le développement régional ». Préoccupé d'épistémologie, il apporte des contributions à une épistémologie de l'agir du chercheur.
\end{abstract}

François Papy (NSS) : Au moment où, collés aux événements, les médias parlent des crises alimentaire et écologique, l'agronomie se trouve projetée sur le devant de la scène. En joignant les connaissances sur des processus biologiques et écologiques aux décisions d'action, elle se trouve au cœur de débats vitaux pour la société humaine. L'histoire récente de cette discipline doit pouvoir, sans nul doute, faire comprendre le rôle qu'elle peut jouer. C'est pourquoi la direction de la revue Natures Sciences Sociétés a souhaité cet entretien avec quelqu'un qui a été à la source de la renaissance de l'agronomie, au milieu du siècle dernier. Avec vous, qui avez été assistant de Stéphane Hénin, je souhaiterais prendre comme point de départ l'enseignement de ce dernier, que beaucoup d'agronomes considèrent comme fondamental. Cela nous permettra de partir de votre propre point

Auteur correspondant : F. Papy, papy.francois@numericable.fr de départ. De quand date votre rencontre avec Stéphane Hénin?

Michel Sebillotte : Venu de l'Inra, « Monsieur Hénin» (c'est ainsi que nous l'appelions entre nous à l'Agro ${ }^{1}$ ) a été nommé, à la fin de 1958, professeur d'agriculture à l'Institut national agronomique. Le directeur de cet établissement, M. Blais, souhaitait recruter un professeur d'agriculture qui en développe une vision suffisamment "théorisante ». Monsieur Hénin est apparu aux membres du jury de concours comme capable de répondre à cet objectif et de faire la jonction entre pratique agricole et aspects "scientifiques ». Durant mon service militaire, j'avais déjà eu l'occasion de le rencontrer. Et lorsqu'il a obtenu deux postes de jeunes assistants en novembre 1961, j'ai été recruté assistant de Monsieur Hénin à la chaire d'agriculture, en même temps que Jean-Pierre Deffontaines.

\footnotetext{
1 Il s'agit de l'Institut national agronomique de Paris.
} 
Sur Monsieur Hénin, il y aurait beaucoup de choses à dire, et d'abord mon admiration pour celui qui est toujours resté mon maître. À l'Inra, avant son recrutement comme professeur à l'Agro, il avait été directeur du Laboratoire des techniques culturales où il animait une jeune équipe. Pour montrer l'intérêt de prendre les techniques culturales comme objet d'étude scientifique, il venait d'écrire son premier ouvrage avec la collaboration de sa jeune équipe de chercheurs. Le Profil cultural : principes de physique du sol a paru en 1960. L'étude du travail du sol l'avait conduit à fréquenter beaucoup de praticiens et à participer à différentes manifestations, comme le concours national de labour. Et l'outil que constituait le profil cultural était pour lui et son équipe un moyen d'investigation très précieux pour discuter, avec les agriculteurs, des effets de leurs pratiques sur le sol. Les jeunes enseignants de la chaire d'agriculture que nous étions ont tout de suite adopté le profil cultural ${ }^{2}$. Pour comprendre ce qui se passait dans une parcelle, nous eûmes, grâce à lui, tout de suite notre outil distinctif.

Je tiens à dire ici combien, grâce à ses qualités scientifiques, dans ses fonctions de professeur d'agriculture, Monsieur Hénin a permis l'éclosion de l'agronomie, lui donnant par anticipation ses lettres de noblesse. Il a ouvert sur le futur une série de pistes, particulièrement à travers différentes notes à l'Académie d'agriculture. Entre autres questions, il se préoccupait beaucoup des conséquences de l'agriculture sur l'environnement.

Pourtant, aujourd'hui, avec du recul, sur les questions de théorisation et de fabrication d'outils pour étudier les champs, on peut se demander si Monsieur Hénin se représentait l'agronomie comme une science ; il la voyait plutôt comme un art.

F.P. : Eh bien, justement, par quel chemin êtes-vous arrivé à considérer et à défendre l'agronomie comme discipline scientifique, et comment avez-vous développé un rapport, visiblement très fort, à l'action, vous qui avez intitulé «Penser et agir en agronome » la préface d'un ouvrage récent d'agronomie, écrit par un certain nombre de vos successeurs?

Michel Sebillotte : Comme vous allez le voir, les chemins ont été multiples. Tout d'abord, je retiens les trois pistes de travail scientifique que Monsieur Hénin m'a suggérées dès mon recrutement; elles lui semblaient correspondre à des questions essentielles dans des secteurs très divers. La première consistait à évaluer si on pouvait utiliser avec profit la recherche opérationnelle pour résoudre un certain nombre de problèmes d'aide à la décision en agriculture. La seconde portait sur les rotations

\footnotetext{
2 Dans l'ouvrage cité plus haut, le profil cultural est défini comme «l'ensemble constitué par la succession des couches de terre, individualisées par l'intervention des instruments de culture, des racines de végétaux et des facteurs naturels réagissant à ces actions ".
}

culturales et sur les monocultures. La troisième consistait à reprendre les travaux de Jonard (chercheur à l'Inra) et à se pencher sur les facteurs de l'élaboration du rendement. Ces trois pistes ont été déterminantes dans la suite de ma carrière scientifique. Mes recherches sur les rotations ${ }^{3}$ se sont concrétisées par le lancement, en 1963, d'une expérimentation de longue durée (21 ans) ; elle a été l'occasion de faire de nombreuses observations qui me conduisirent à jeter les jalons de ce qui deviendra l'élaboration du rendement comme pivot de l'analyse d'une parcelle.

À la même époque, le directeur de l'Agro me confiait la direction de la nouvelle ferme de l'école, à Palaiseau. Ceci eut une grande importance pour moi, en prolongeant mon expérience tunisienne et en me formant à l'action. J'avais déjà retenu beaucoup de l'expérience de mon père, colon dans le Sud tunisien. Je l'avais vu innover, chercher de nouvelles techniques, de nouveaux débouchés. Et j'avais même envisagé de lui succéder avant de préparer l'Agro. C'est dire combien la direction d'une ferme correspondait à mon goût pour l'action.

Enfin, Monsieur Hénin m'a fait découvrir Gaston Bachelard et l'épistémologie.

Ces différents aspects ont joué un rôle essentiel dans ma formation. Ils m'ont permis de rapprocher théorie et pratique. À titre d'exemple, cela m'a servi dans la responsabilité de l'exploitation de l'Agro, où j'ai essayé d'exploiter les observations que j'y faisais chaque semaine sur toutes les parcelles, comme, par exemple, l'apparition, un beau matin, du rougeoiement des pieds de blé. Les situations culturales évoluaient; mais comment les observer et que tirer de ces visites régulières? Cela m'a donné l'idée de formaliser, sous le nom de «tour de plaine », les observations qu'un agronome peut faire en parcourant, à intervalles réguliers, l'ensemble des parcelles d'une exploitation. La méthode s'est avérée suffisamment pertinente pour que je puisse en tirer des publications sur des cultures sensibles aux états structuraux du sol.

Durant cette période, je me suis ouvert à d'autres situations agricoles : la forêt tropicale ivoirienne, les terrains de parcours du Maroc oriental... D'ailleurs, j'ai aussi contribué, dans ce pays, à développer l'enseignement de ce qui, pour moi, s'appelait résolument l'agronomie. Mon souci constant était de faire servir chacune de mes expériences au reste de ce que je faisais : ainsi, au Maroc, je cherchai à voir à quoi pouvait servir ce qui se faisait en France, et réciproquement.

Dans l'enseignement, j'ai cherché à faire passer, à la fois, des données théoriques et pratiques, en donnant, notamment, un poids important au stage en exploitation. J'ai contribué à étendre le stage de première année sur trois périodes, soit onze semaines au total, ce qui a permis d'en faire un vrai outil de formation. Mon objectif

\footnotetext{
${ }^{3}$ On appelle rotation une succession de cycles culturaux d'espèces ou $d^{\prime}$ associations $d^{\prime}$ 'espèces qui se répète au cours $d u$ temps.
} 
était de rendre les étudiants capables de mobiliser les savoirs acquis en agronomie pour questionner la réalité agricole (celle de leur exploitation de stage et de sa région agricole), quand leur tendance était d'être des imitateurs de leur maître de stage, des reproducteurs passifs de ses savoirs. Mais j'ai cherché également à initier les étudiants à l'activité de recherche par des travaux personnels et à développer le goût de l'épistémologie par une lecture commentée de l'ouvrage de Bachelard intitulé La Formation de l'esprit scientifique.

Monsieur Hénin m'a, de fait, accompagné jusqu'en 1966, date à laquelle il est retourné à l'Inra. Ensuite, il a bien fallu que je sois autonome, ce qui m'a amené à prendre quelques distances, tout en cherchant à prolonger son œuvre. C'est ainsi que j'ai été conduit à définir l'agronomie comme une discipline se rattachant aux sciences de la nature, à partir des activités et des réflexions que j'avais eues jusqu'alors.

F.P. : Cette conception de l'agronomie, vous la publiez alors dans un article fondateur, paru en 1974 dans les Cahiers ORSTOM. Il est intitulé « Agronomie et agriculture. Essai d'analyse des tâches de l'agronome ${ }^{4}$ ». On y trouve à la fois une discipline, s'identifiant autour d'objets d'étude et de concepts, et des hommes d'action : les agronomes, qui contribuent à l'élaborer dans leurs actions mêmes. Quelles sont les idées principales que vous y développiez?

Michel Sebillotte : Je vais répondre à la question en l'enrichissant d'idées exprimées plus récemment. Pour moi, l'activité scientifique du chercheur (soutien de celles du formateur et de ceux qui utilisent l'agronomie) consiste à considérer qu'une discipline se définit par ses objets d'étude abordés à partir de concepts et de théories. En 1974, l'agronomie n'avait pas de concept récent ; les agronomes travaillaient certes dans des parcelles culturales d'agriculteurs ou des parcelles de dispositifs expérimentaux, mais ne disposaient que d'ébauches de méthodes, en dehors des méthodes statistiques! Ils étudiaient d'ailleurs des stations plus que des parcelles, dominés qu'ils étaient par l'idée d'éviter l'hétérogénéité. Il fallait suivre des protocoles expérimentaux exigeants pour en réduire les effets perturbateurs, par exemple pour établir des relations entre techniques culturales et production : le fameux rendement! L'hétérogénéité était gênante parce qu'il y avait opposition entre une conception dominante de la science, visant à l'universel, et une autre, plus contextuelle, différenciant les situations, permettant de les classer et de raisonner. Dans cette optique, j'essaye de montrer que l'agronome est constamment en train de fabriquer l'agronomie, dont le lieu d'application est l'agriculture. L'agronome se trouve ainsi en tension entre un rôle consistant à fabriquer l'agronomie et

\footnotetext{
4 Cahiers ORSTOM, série Biologie, 24, 3-25 (http :// www.ird.fr/bondy/tdp/biologie/index_auteurs/auteur_S.htm).
}

un autre lui imposant de participer à l'amélioration de l'agriculture. Il a donc des tâches dans les domaines de l'agronomie et de l'agriculture que, dès 1974, je distingue clairement sans les séparer. Je propose, dans cet article, le concept d'itinéraire technique défini comme « une combinaison logique et ordonnée de techniques qui permettent de contrôler le milieu et d'en tirer une production donnée ». Il est bien entendu qu'il existe plusieurs stratégies possibles pour atteindre un objectif donné et que, au cours du temps, en fonction des circonstances, des adaptations tactiques sont possibles. L'agronome doit concevoir des itinéraires techniques, préciser les conditions dans lesquelles les mettre en œuvre, en évaluer le coût. Mais, très vite, ce concept sert aussi à analyser les pratiques des agriculteurs. Cet agronome, celui de la parcelle et de l'itinéraire technique, je le nomme aujourd'hui agronome $\mathrm{n}^{\circ} 1$. En 1974, je n'avais pas les instruments pour aborder ce qui deviendra mon deuxième objet d'étude : $l^{\prime}$ agriculteur en train de cultiver, celui de l'agronome $n^{\circ} 2$. Plus tard, le territoire apparaîtra comme le troisième objet, celui de $1^{\prime}$ agronome $n^{\circ} 3$.

C'est la réflexion épistémologique qui m'a «imposé » de définir l'objet «parcelle», avec les concepts et les méthodes appropriés. Mais alors les agronomes que nous étions se sont trouvés comme enfermés dans cet objet, oscillant entre une attirance pour les sciences physiques et chimiques, ou pour les sciences du vivant. Nous savions certes qu'existaient entre les parcelles des échanges de particules, de molécules, de gènes, mais nous n'avions pas encore d'outils pour les étudier. Nous savions aussi le rôle de l'agriculteur dans les choix techniques, mais, à cette époque, l'agriculteur n'était objet d'étude que pour les sciences humaines!

F.P. : Voilà que vous allez sortir du strict champ des sciences de la nature et que vous allez faire que l'agriculteur devienne également un objet d'étude des agronomes. Il fallait une certaine audace dans un contexte académique qui maintenait (et maintient toujours) une dichotomie entre sciences de la nature et sciences sociales et ne faisait guère de place aux disciplines qui portent sur les techniques et les pratiques. Votre définition d'un deuxième objet d'étude (l'agriculteur en train de cultiver) date des années 1980. Approfondissons maintenant cette étape.

Michel Sebillotte : J'ai découvert, à la fin des années 1970, la psychologie ergonomique, qui m’a donné des outils pour étudier, en agronome, l'agriculteur. Cela m'a permis de confirmer l'idée que l'agriculteur avait "de bonnes raisons de faire ce qu'il faisait », comme il était courant de l'affirmer dans le département Systèmes agraires et développement de l'Inra. Bonnes au regard de sa conception, bien sûr. Il fallait donc avoir accès à cette conception. Profitant d'une collaboration avec les agriculteurs de la région de Noyon, en Picardie, j'ai essayé de 
comprendre ce qu'était l'activité de l'agriculteur dans la gestion de ses champs cultivés. Avec l'aide d'étudiants, puis de jeunes collaborateurs, j'ai progressivement mis au point ce qui allait devenir «le modèle de l'agriculteur pour l'action », qui s'est révélé avoir une portée extrêmement générale. On a ainsi mis au jour le processus mental (et social) qui structure l'action des agriculteurs pour cultiver leurs parcelles. Ces modèles sont des formalismes simplifiés des processus qui permettent de reproduire les actions d'un agriculteur dans la gestion de ses champs. Ils sont constitués des objectifs à atteindre avec un certain nombre de règles à appliquer et d'indicateurs à suivre ; les règles prévoient ce qu'il faut faire aussi dans le cas où les conditions, entre autres climatiques, ne permettent pas de suivre le calendrier de travail initialement prévu. Ces modèles comportent ainsi des solutions de « rattrapage », ce qui est particulièrement intéressant. $C^{\prime}$ est cet ensemble qui permet aux agriculteurs de cultiver ; ils ont tous dans la tête un modèle pour l'action, fruit de leur apprentissage, individuel et social, au fil des années. Ce modèle est la réponse élaborée pour obtenir, avec un maximum de chances, la combinaison optimale des techniques culturales. L'agriculteur tente ainsi de se prémunir contre une non-réalisation ou une réalisation en conditions désastreuses; il cherche une quasi-certitude, une fois fixés pour une série de champs des objectifs de production.

Mettre en évidence un modèle pour l'action exige d'appliquer son esprit critique à ce que dit l'agriculteur, qui, quelles que soient les précautions prises, a tendance à répondre ce qu'il croit que l'enquêteur attend de lui. Le concept de modèle pour l'action ne découle pas d'un simple discours d'agriculteur; il n'a pu émerger que parce que, en même temps que nous faisions parler l'agriculteur, nous l'observions sur ses parcelles et nous faisions des enregistrements sur les états du sol et des cultures que nous savions interpréter. Ainsi, les enquêtes auprès d'agriculteurs afin de déterminer leurs modèles pour l'action nécessitent une collaboration étroite entre les compétences des agronomes $n^{\circ} 1$ et $n^{\circ} 2$, qui se distinguent parfois en différents métiers. C'est une démarche spécifique d'agronomes. Dans la filiation de ce concept, des modélisations de règles de décision ont été proposées et ont été couplées à des modèles de culture, ce qui, dans les colloques internationaux, constitue une démarche originale des agronomes français pour concevoir de nouveaux procédés de culture.

F.P. : Fin 1991, Guy Paillotin, alors président de l'Inra, vous sollicite pour une mission de prospective : il vous demande de proposer des orientations pour son institution. Cela a constitué le point de départ d'une nouvelle étape et l'occasion, pour vous, d'élargir votre champ de réflexion ainsi que les collaborations avec d'autres scientifiques et d'autres partenaires sociaux.
Michel Sebillotte : Quelles pourraient être les évolutions de l'agriculture? Que devait faire l'Inra pour y répondre au mieux? Voilà les questions posées par Guy Paillotin. La réflexion m'a pris une année pleine. J'ai constaté que l'Inra s'éloignait de plus en plus des préoccupations des agriculteurs. Avec l'équipe que je dirigeais, j'ai fait un diagnostic d'ensemble sur l'état de l'agriculture qui m'a permis de faire des propositions en rajoutant aux orientations de recherche de l'Inra quatre nouveaux axes : la gestion du vivant; la qualité des produits agricoles; la gestion de l'environnement écologique ; le développement régional et la gestion de l'espace rural. Ces recherches devaient être conduites en concevant l'innovation comme processus social, c'est-à-dire avec le souci impérieux de revenir au réel des acteurs. Ce rapport a entraîné mon détachement à l'Inra en $1993^{5}$. Dans un service nouveau, j'ai d'abord mis en place un programme national de recherche en partenariat «Pour et sur le développement régional » (PSDR). Des travaux «sur» le développement existaient déjà ; ils consistaient à juger si on avait abouti aux objectifs désirés à partir de l'analyse de ce qui s'était passé ; il fallait poursuivre dans cette voie. C'est le «pour» qui posait question : comment la recherche pouvait-elle s'impliquer dans quelque chose qui était le développement du territoire, à savoir un lieu de construction et de mise en œuvre de projets en partenariat avec l'ensemble des acteurs impliqués, au-delà même de l'agriculture au sens large? Comment construire ensemble des questions, des listes de problèmes? Comment, ensuite, transformer ces problèmes en thèmes de recherche et comment communiquer? C'est ce quej'ai appelé, récemment, « la boucle vertueuse de la recherche finalisée». Une fois établie, avec les partenaires, une liste de questions à résoudre, c'est en réunissant des chercheurs de différentes disciplines qu'il est possible de transformer ces questions de la pratique en questions pouvant être traitées scientifiquement, car il n'a jamais été question pour moi de ne pas faire de science. Cela m'a conduit à dégager justement le territoire comme objet transdisciplinaire, n'appartenant à aucune discipline, sachant que chaque discipline ne peut pas aborder l'ensemble des problèmes d'un projet de développement d'un territoire. L'agronome peut et doit aborder la dimension spatiale de ses problèmes, ce qui revient dans une conception systémique à considérer la parcelle ou l'ensemble de parcelles comme un système ouvert. Mais le territoire d'une communauté humaine, qui résulte des activités d'un grand nombre d'acteurs, exige, pour le comprendre, une approche transdisciplinaire. C'est pour moi l'occasion de définir le troisième objet de l'agronomie : le territoire, lieu d'insertion de la parcelle, mais surtout lieu pour

5 L'esssentiel de ce rapport a été repris dans Sebillotte, M., 1996. Les Mondes de l'agriculture: une recherche pour demain, Paris, INRA Éditions. 
l'agronome de son implication dans les problèmes de développement. C'est le métier de l'agronome $\mathrm{n}^{\circ} 3$.

F.P. : Vous élargissez donc, une fois encore, le champ d'activité que vous proposez aux agronomes à travers les objets d'étude successivement construits : la parcelle ; l'agriculteur cultivant ses parcelles; le territoire, lieu de construction collective de projet de développement. Examinons maintenant si ces objets, les concepts et théories qui les accompagnent, permettent à l'agronomie de relever le défi soulevé par la convergence des crises alimentaire et écologique. On entend certains parler d'une nécessaire « refondation » de l'agronomie. Qu'en pensezvous?

Michel Sebillotte : Si les agronomes n'ont pas forcément encore tous les concepts pour théoriser tous les aspects de leur science, ils ont déjà suffisamment de choses pour agir. Mais il ne faut surtout pas confondre les agronomes et l'agronomie qu'ils ont construite avec un modèle particulier d'agriculture. Je crois que les agronomes se sont laissés enfermer dans une telle confusion. Comme la société incitait à développer une agriculture intensive, les agronomes ont développé les concepts et théories qui permettaient de faire face à cette demande sociale. Mais, à aucun moment, la science «agronomie » n'a considéré l'agriculture intensive et ses options comme la seule voie possible. Monsieur Hénin, en 1980, dans un rapport devenu célèbre ${ }^{6}$, a dénoncé l'effet des activités agricoles sur la pollution des eaux par les nitrates. Dans sa lignée, j'ai participé de façon active à l'opération nationale "Ferti-Mieux », qui avait pour objectif explicite de lutter contre cette pollution. Si l'on en revient aux fondements de l'agronomie, je pense que les trois agronomes $\left(n^{\circ} 1, n^{\circ} 2\right.$ et $\left.n^{\circ} 3\right)$ ont les concepts théoriques pour répondre largement à une grande partie des questions d'aujourd'hui. Par exemple, la recherche par certains du rendement maximum, en agriculture intensive, n'est jamais considérée comme un objectif en agronomie : c'est une référence, l'expression d'une potentialité, qui fixe l'échelle sur laquelle situer les rendements qu'il serait possible de viser. Lorsque l'on reproche aux agriculteurs d'être des "pollueurs ", là encore l'agronomie fournit l'armature conceptuelle et théorique pour discuter sérieusement du choix d'une pratique de fertilisation qui ne soit pas polluante. C'est que l'agronomie telle que nous l'avons pratiquée n'a jamais été une mise en relation directe entre techniques culturales et rendement, ou encore entre une culture donnée et le rendement de la suivante. Elle consiste à étudier comment les opérations techniques modifient les processus bio-physicochimiques au sein du champ cultivé et aboutissent à une production et un état final du champ donnés. Aussi la

6 Hénin, S., 1980. Rapport du groupe de travail Activités agricoles et qualité des eaux, réalisé pour les ministères de l'Agriculture et de l'Environnement. (Rapport ronéoté.) théorie agronomique permet-elle d'associer à des procédés de culture une production et un bilan de l'état des ressources utilisées et du milieu. Redisons-le, nous disposons déjà de beaucoup de concepts et théories pour agir : mettons-les en pratique véritablement et tironsen les conséquences socioéconomiques! Il n'y a pas lieu d'estimer nécessaire une " refondation ».

Cependant, la science « agronomie» doit encore et toujours progresser! Par exemple, pour affronter certains des nouveaux problèmes qui se posent et auxquels les agronomes d'aujourd'hui sont insuffisamment préparés. Revenons au territoire. Une de ses caractéristiques est d'être soumis à des exigences contradictoires auxquelles il faudrait répondre. Sur ce point, à mon avis, la recherche est très sollicitée et beaucoup d'investigations devraient être entreprises pour aider la formulation de compromis. Comment faire? Il faut apprendre à hiérarchiser des données incommensurables. Cette hiérarchisation ne peut être traitée du seul point de vue scientifique ; elle ne peut se faire sans l'accord des habitants de ce territoire sur un modèle de développement, donc sur le type de compromis à organiser, de hiérarchie à promouvoir... Cela, on ne sait pas bien le faire aujourd'hui; la coconstruction est un domaine dans lequel les chercheurs ont du mal à s'engager : ils vont devoir changer!

F.P. : Voilà bien une question cruciale : celle de l'engagement des chercheurs auprès de partenaires sociaux pour formuler des problèmes et rechercher des compromis. Suffit-il que les chercheurs, s'ouvrant aux partenaires, mettent leur science à la disposition d'acteurs considérés comme légitimes pour établir les compromis à faire?

Michel Sebillotte : Non, ce n'est pas suffisant. J'insisterai d'abord sur la nécessité de réflexions d'ordre épistémologique : que fait-on lorsque l'on agit et pense en agronome? On souligne, à juste titre, la fécondité des réflexions aux interfaces. Je voudrais cependant insister à nouveau sur ce qu'on entend par discipline. Pour moi, je l'ai dit, l'agronomie est constituée de la réunion de trois métiers différents, donc de trois pratiques : celles des agronomes $n^{\circ} 1, n^{\circ} 2$ et $n^{\circ} 3$; mais ils doivent être en dialogue serré et permanent. Comment, en effet, comprendre la parcelle sans une connaissance approfondie de l'agriculteur en train de cultiver, ou comment participer au développement d'un territoire si l'on n'a pas une idée fine des outils agronomiques disponibles pour l'agriculteur? Je pense que les trois types d'agronome ne dialoguent pas assez entre eux. Chacun des trois a, en dehors de sa discipline, son propre réseau d'interdisciplinarité (selon les cas avec les sciences biophysiques, biologiques, sociales) et il n'exerce bien son métier d'agronome que s'il est capable d'avoir un langage commun avec son réseau. De ce point de vue, les agronomes doivent beaucoup plus se cultiver et réfléchir sur ce qu'ils pensent et font en tant qu'agronomes! 
Ce n'est pas suffisant non plus parce que les questions soulevées plus haut contiennent de façon sous-jacente celles du choix de société et de développement auxquels on accepte de participer. Apparaît alors l'agronome qui voudrait être un «militant critique ». À côté des aspects scientifiques, il devrait faire intervenir une dimension éthique pour laquelle il serait prêt à se battre. On en a une bonne illustration à travers la question des organismes génétiquement modifiés et la position de chercheurs qui essayent, justement par militantisme critique, de ne pas mettre leur science au service d'un projet qu'ils n'auraient pas, par ailleurs, discuté en tant que citoyens.

F.P. : J'ai commencé en évoquant les crises alimentaire et écologique comme justification de cet entretien. Maintenant, avant de le finir, je souhaiterais vous demander comment vous voyez les possibilités de résoudre l'antagonisme entre une demande alimentaire qui croît et la nécessité dans laquelle nous sommes d'économiser des ressources limitées et de préserver la viabilité de l'écosystème terrestre.

Michel Sebillotte : Je vois deux «limites techniques » pour relever à terme le défi soulevé par la convergence des crises alimentaire et écologique. D'une part, sera-t-on capable de produire suffisamment pour nourrir un nombre croissant d'habitants de la planète? Je m'interroge. Je ne crois guère aux espoirs que fondent certains sur les terres encore à cultiver, à irriguer, etc. Je pense que les questions des modèles de consommation et du nombre de consommateurs sont capitales et doivent être posées. D'autre part, concernant l'environnement, je doute que l'on puisse éviter de se poser la question des effets de la préservation de l'environnement sur le niveau de production et donc sur le degré de préservation que l'on souhaite. Je doute, en effet, que l'on puisse facilement résoudre les problèmes par l'emploi de techniques nouvelles (commel'agriculture de précision), par la généralisation, par exemple, de « l'agriculture biologique »... Certes, la recherche peut faire beaucoup de choses, entre autres sur le plan technique; mais à quel rythme, dans quelles proportions? Et, surtout, que diront les sociétés des solutions proposées? Les sociétés sont globales et l'agriculture n'est qu'un de leurs composants!

Comment, concrètement, faire vivre ce « militantisme critique »? Je conclurai en disant, d'abord, qu'il me semble que les agronomes, s'ils restent des hommes de terrain, capables d'utiliser leurs connaissances pour émettre des diagnostics, faire des pronostics et les tester, peuvent beaucoup apporter aux sociétés modernes. Mais ils doivent rester des hommes d'action ancrés dans le réel. N'est-ce pas, entre autres, l'une des raisons d'être de l'agronome $n^{\circ} 3$ ? S'il sait entretenir un dialogue avec ses homologues des autres disciplines, il peut donner tout son rôle à la science dans la vie des sociétés ; il peut, jouant également un dialogue avec les "profanes", construire avec eux de nouvelles sociétés plus justes et plus respectueuses de l'environnement, en un mot bâtir un développement durable. 